\title{
Editorial: Hair Follicle Stem Cell Regeneration in Aging
}

\author{
Mingxing Lei ${ }^{1,2 *}$, Sung-Jan Lin ${ }^{3,4}$ and Cheng-Ming Chuong ${ }^{5}$ \\ 1111 Project Laboratory of Biomechanics and Tissue Repair, College of Bioengineering, Chongqing University, Chongqing, \\ China, ${ }^{2}$ Key Laboratory of Biorheological Science and Technology of the Ministry of Education, College of Bioengineering, \\ Chongqing University, Chongqing, China, ${ }^{3}$ Department of Biomedical Engineering and Department of Dermatology, College of \\ Engineering and College of Medicine, National Taiwan University, Taipei, Taiwan, ${ }^{4}$ Department of Dermatology, National Taiwan \\ University Hospital, Taipei, Taiwan, ${ }^{5}$ Department of Pathology, Keck School of Medicine, University of Southern California, Los \\ Angeles, CA, United States
}

Keywords: aging, hair regeneration, niche, skin organoid, wound-induced hair neogenesis

\section{Editorial on the Research Topic}

\section{Hair Follicle Stem Cell Regeneration in Aging}

With stem cells that can be activated and silenced cyclically, hair follicle experiences multiple rounds of growth phase (anagen), regression phase (catagen), and resting phase (telogen) during lifespan. Hair cycling is initiated by cyclic renewal or physiological cyclic regeneration of stem cells. However, tissues and organs undergo structural and functional declines in the aging process, with physiological and pathological changes regulated by intrinsic and extrinsic factors that dictate the cell fate (Lei and Chuong, 2016) (Figure 1). As one of the important appendages of the skin, the hair follicle is a complex mini-organ with visible signs, such as decreased regenerative ability that leads to alopecia, and hair graying due to less melanin production by melanocyte stem cells during aging (Qiu et al., 2019). Hair regenerative ability is gradually decreased because hair follicle stem cells enter a long quiescent state (Chen et al., 2014), or differentiate into other skin epithelial lineages (Matsumura et al., 2016), or escape from the hair follicle niche during aging (Zhang C. et al., 2021). These features promote hair follicle to become a widely used model for studying regeneration. As over $30 \%$ of the population all over the world suffer from partial or complete hair loss, particularly most people undergo alopecia during aging, understanding the mechanism by which hair follicle changes during aging is of great interest in regenerative biology and is essential for regenerative medicine.

The field of hair follicle biology has expanded tremendously with broader scales and diverse approaches that aim to elucidate the mechanisms of hair follicle regeneration, as well as to devise potential therapeutic strategies to delay the aging processes or restore the regenerative ability. Here we organize this Research Topic with a collection of original research and review articles that explore aging-related hair follicle stem cell regeneration. Although not fully covering the comprehensive hair follicle stem cell field, this issue provides new insight into hair regeneration from different perspectives, mainly at the physiological, wound healing, and in vitro cultivation levels.

Skin which covers the surface of our human body is constantly subjected to environmental insults. Ultraviolet (UV) radiation is the major cause of skin aging that is called photoaging, which provokes oxidative stress, inflammatory responses, and DNA damage. Three papers in this Research Topic focus on studying the role of UVA in skin aging (Wang et al., 2020; Bai et al., 2021; Karisma et al., 2021). Bai et al. investigated rapamycin as a macrolide immunosuppressant that protects skin fibroblasts from UVA-induced photoaging (Bai et al., 2021). They found that rapamycin-treated human dermal fibroblasts have a decreased expression of p53 and phosphorylated HSP27, as well as reduced genotoxic and oxidative stress levels. Wang et al. identified purified vitexin compound 1 as a novel lignanoid that attenuates UVA-induced senescence in human dermal fibroblasts in vitro and 
mouse skin in vivo, through repressing UVA-induced phosphorylation of MAPK1 (Wang et al., 2020). Although UVA has a harmful effect to cause skin photoaging, it is a double-edged sword that can be used as a stimulus to switch the photo-controlled drug release upon a "switch on-switch off" procedure, which can be developed for clinical use. A review comprehensively summarized how UVA-triggered drug release as a promising potential for skin photoprotection and phototherapy (Karisma et al., 2021). In addition to UVAinduced photoaging, recent advancements suggest that tissue mechanics is largely involved in regulating skin aging. Another review by Harn et al. described the manifestations of the aging skin, and fully summarized how tissue mechanics influences extracellular matrix dysregulation, wrinkling, and wound healing during skin aging (Harn et al., 2021). They also proposed how perturbed tensional homeostasis impacts physiological functioning of the skin, including how skin tension influences hair regeneration and wound healing. Then, they enumerated several potential chemical and mechanical approaches that may be used for developing new drugs against skin aging.

Hair follicle is one of the skin appendages that is mostly influenced by aging. Hairs are gradually lost during the aging process, which is largely controlled by intrinsic epigenetic/genetic status and extrinsic environmental stimuli that regulate the hair follicle stem cell behavior during hair cycling. Decreased expression of activators and increased expression of inhibitors lead to decreased or failed hair regeneration in aged skin, which may cause alopecia eventually. The telogen phase of the hair follicle, particularly the refractory telogen phase becomes longer and longer, compared to the competent telogen phase during aging. In this Research Topic, Suo et al. identified that different levels of Vitamin A have different roles in regulating hair cycling. High-level of dietary retinyl esters results in a greater percentage of hair follicles in refractory telogen, and vice versa (Suo et al., 2021). They showed that Vitamin A regulates hair follicle stem cell activity through BMP and Wnt signaling pathways. This study identified a new intrinsic factor that regulates hair follicle stem cell activity. Two reviews summarized the recent progress on how intrinsic and extrinsic factors modulate hair follicle stem cells activities. Daszczuk et al. discussed the current views on the intrinsic molecular mechanisms that regulate cyclic hair regeneration (Daszczuk et al., 2020). They proposed an intrinsic oscillation of gene networks model in control of hair cycling, which has potential instruction for translational regenerative medicine. Morgun and Vorotelyak reviewed how inflammation as one of the extrinsic factors that regulates hair cycling, re-epithelialization, and wound-induced hair neogenesis (Morgun and Vorotelyak, 2020). They also discussed how inflammation influences hair follicle stem cell activation during wound healing at the cellular and molecular levels. These two reviews developed further the concept that synergistic action of intrinsic and extrinsic factors is essential for proper hair regeneration.

Dermal papilla (DP) is the signaling center for regulating hair growth and regeneration. In this Research Topic, comparative transcriptome analyses by Weber et al. identified a Wnt agonist,
R-spondin-1, is significantly decreased in the DP of adult human hair follicles compared to that of the fetal scalp hair follicles (Weber et al., 2020). Adult cells which lose the regenerative ability can be partially restored to regenerate hair follicles with addition of R-spondin-1 recombinant protein. Another research by $\mathrm{Yu}$ et al. showed that Twist1 functions on regulating hair follicle induction ability in the DP by forming a ternary complex with Tcf 4 and b-catenin, which delays the aging process of the DP cells by Tcf4-mediated Wnt/b-catenin signaling pathway (Yu et al., 2020). Under pathological conditions such as androgenetic alopecia (AGA) which is occurring in more than half of men aged over 50 years old, hair follicles are attacked by dihydrotestosterone and then become miniaturized. Liu et al. identified that Zyxin, an actin-interacting protein, is increased in the DP of AGA-affected frontal hair follicles compared to that of the unaffected occipital hair follicles (Liu et al., 2020). Knockout of Zyxin leads to enhanced hair growth and anagen reentry, indicating that Zyxin is an inhibitor for hair regeneration. This offers a potential therapeutic target for treating AGA.

As skin is located at the outmost of our body, it is vulnerable to be injured by mechanical, chemical, or biological insults. Research conducted by Yang et al. used a promising new method called photodynamic therapy to treat mouse skin wounds infected with Pseudomonas aeruginosa (Yang et al., 2020). They showed that 5-aminolevulinic acid photodynamic therapy is beneficial for eliminating Pseudomonas aeruginosa and accelerating skin wound healing in mice, by modulating the expression of inflammatory factors (TNF-a and IL-1b) and growth factors (TGF-b1 and VEGF). Since the establishment of the wound-induced hair neogenesis model by Ito et al., in 2007 (Chuong, 2007; Ito et al., 2007), a number of following studies aimed to study the molecular mechanisms by which de novo hairs regenerate at the center of the wound bed during healing of large cutaneous wounds. Bhoopalam in this Research Topic reviewed the recent progress in digging the underpinnings of woundinduced hair neogenesis, at the cellular (hair follicle stem cells, fibroblasts, inflammatory cells) and molecular (Wnt/b-catenin, Hedgehog, dsRNA/IL-6/STAT3/Retinoic Acid, Hippo signaling pathways) levels (Bhoopalam et al., 2020). They also proposed several wonderful interesting questions that remain to be further investigated in this field. Besides, this issue also included one research paper which identified IL-36a as a new factor modulating the outcome of wound-induced hair neogenesis (Gong et al., 2020). They show that IL-36a is increased during wound healing, and application of recombinant murine IL-36a protein into large skin wound promotes wound-induced hair neogenesis, probably through regulating the IL-6/STAT3 pathway.

Scientists also have been trying to restore hair regeneration using in vitro culture models, with focuses on modulating hair follicle stem cells and DP cells. Bak et al. in this Research Topic transplanted the cultured human outer root sheath (ORS) cells along with freshly isolated neonatal mouse dermal cells to the nude mice, and observed that the long-term (42 days) cultured ORS cells have a decreased trichogenicity compared to the shortterm (20 days) cultured ORS cells (Bak et al., 2020). They identified that FOXA2 in ORS cells functions in regulating the 


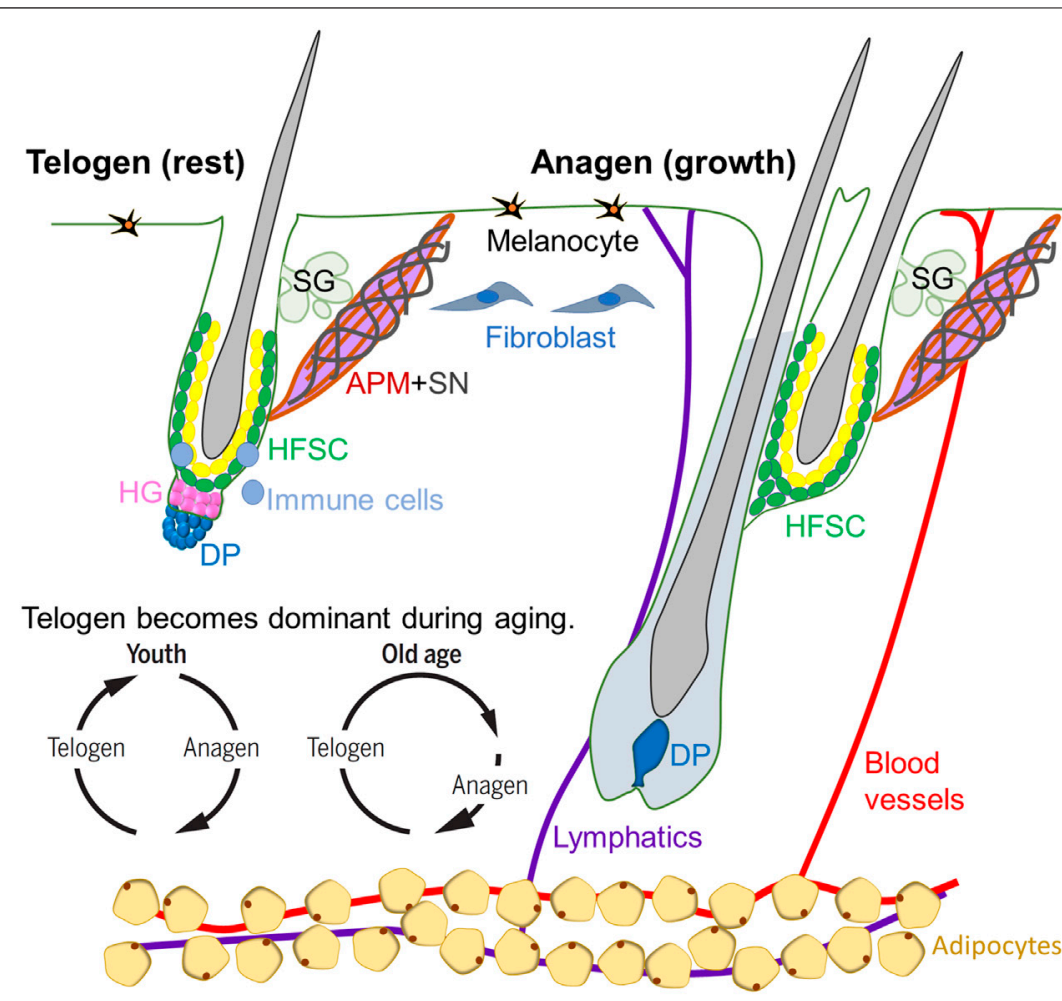

\begin{tabular}{|c|c|c|}
\hline Stem cell & Niche & Environment \\
\hline $\begin{array}{l}\text { (Daszczuk et al., 2020; } \\
\text { Suo et al., 2021) }\end{array}$ & $\begin{array}{l}\text { (Liu et al., 2020; Morgun } \\
\text { and Vorotelyak, 2020; } \\
\text { Weber et al., 2020; Yu et } \\
\text { al., 2020) }\end{array}$ & $\begin{array}{l}\text { (Wang et al., 2020; Bai } \\
\text { et al., 2021; Harn et al., } \\
\text { 2021; Karisma et al., } \\
\text { 2021) }\end{array}$ \\
\hline WIHN & \multicolumn{2}{|c|}{ Culture model } \\
\hline $\begin{array}{l}\text { (Bhoopalam et al., 2020; } \\
\text { Gong et al., 2020; Yang } \\
\text { et al., 2020) }\end{array}$ & \multicolumn{2}{|c|}{$\begin{array}{l}\text { (Bak et al., 2020; Chen et al., 2020; Jang et al., } \\
\text { 2020; Qiu et al., 2020; Fukuyama et al., 2021; } \\
\text { Wen et al., 2021; Zhang et al., 2021b) }\end{array}$} \\
\hline
\end{tabular}

FIGURE 1 | Hair follicle stem cell regeneration in aging. Hair follicle undergoes cyclic renewal, which is regulated by cyclic activation of quiescent stem cells controlled by a core circuit involving beta-catenin. This core molecular circuit involves interactions between hair follicle stem cell (HFSC) and dermal papilla within the hair follicle. Adjacent to the follicles, blood vessels, nerves, arrector pili muscle, intradermal adipose tissues, etc. form a larger niche that also can affect HFSC activation. Further, outside environment can also modulate the cyclic renewal behavior of hair follicles. The effects of these modulators change during aging. The telogen phase of the hair cycle becomes dominant during aging, leading to decreased hair regeneration. Aged cells can be reactivated to regenerate hair follicles under wound-induced hair neogenesis condition or using several 2D or 3D culture models. APM, arrector pili muscle; DP, dermal papilla; HFSC, hair follicle stem cells; HG, hair germ; SG, sebaceous gland; SN, sympathetic nerve.

trichogenicity of human ORS cells. Wen et al. revealed that inhibition of Rho-Associated Protein Kinase by a specific inhibitor Y-27632 can increase the adhesion, proliferation, and stemness of the primary cultured human hair follicle stem cells through the ERK/MAPK pathway (Wen et al., 2021). As twodimensional (2D) cultured cells quickly lose their regenerative ability, most researches turned to modulate hair regeneration under 3D culture conditions. Zhang et al. compared the gene expression in $2 \mathrm{D}$ and $3 \mathrm{D}$ cultured $\mathrm{DP}$ cells treated with dihydrotestosterone, which can inhibit proliferation but induce apoptosis in DP cells co-cultured with ORS cells (Zhang Y. et al., 2021). Without dihydrotestosterone treatment, the ORS cells showed significant proliferation when co-cultured with DP cells. They used RNA-sequencing to identify that extracellular matrix synthesis is increased in 3D culture DP cells, and set up a transcription factor-miRNA coregulatory network in this coculture system, which may be beneficial for future AGA studies at the molecular level. In addition to the extracellular matrix, the same group also identified that miR-140-5p in small extracellular vesicles secreted by DP cells suppresses Bmp2 expression to promote proliferation of ORS and hair matrix cells in a coculture model (Chen et al., 2020). Tissue engineering models have been established to promote hair follicle neogenesis. One research article by Fukuyama et al. set up a reconstitution assay, in which 
human-induced pluripotent stem cell-derived DP cell aggregates were co-cultured with human keratinocytes in a $3 \mathrm{D}$ cylindrical structure inserted with a fragment of guide nylon fiber (a mimic hair fiber). They observed morphologically resembled hair follicle structure that expresses hair follicle-related genes in the 2-week culture (Fukuyama et al., 2021). This study adds a new tissue engineering model for hair regeneration, based on the principle of epithelial-mesenchymal interaction.

In vitro culture models have been established for drug screening. Jang et al. set up a 3D co-culture system, in which human DP cells and ORS cells were cultured in an ultra-low attachment plate, and formed a "two-cell assemblage" structure that can be used as a model to test the hair growth-promoting effect of library molecules (Jang et al., 2020). Epithelialmesenchymal interaction also leads to the formation of a skin cyst when mouse epidermal and dermal cells were mixed and 3dimensionally cultured on a transwell insert, namely organoid culture (Lei et al., 2017). The dissociated single cells can selforganize and undergo a series of morphological transitions, forming a planar hair-bearing skin through aggregation, polarization, coalescence, and planarization stages. Compared to the clinically diagnosed skin cyst, we proposed that the skin cyst forming during skin organoid culture is not a pathological dead-end, but harbors the potential to regenerate skin appendages such as hair follicle and sebaceous gland (Qiu et al., 2020).

In conclusion, recent understanding of aging includes stem cell exhaustion, cellular senescence, altered intercellular communication, epigenetic alterations, genomic instability, telomere attrition, loss of proteostasis, deregulated nutrient sensing, mitochondrial dysfunction, environmental stresses, etc. These changes lead to obvious features in the skin such as hair loss and graying, skin wrinkling, age spots, loss of elasticity,

\section{REFERENCES}

Bai, G.-L., Wang, P., Huang, X., Wang, Z.-Y., Cao, D., Liu, C., et al. (2021). Rapamycin Protects Skin Fibroblasts from UVA-Induced Photoaging by Inhibition of P53 and Phosphorylated HSP27. Front. Cel Dev. Biol. 9, 633331. doi:10.3389/fcell.2021.633331

Bak, S.-S., Park, J. M., Oh, J. W., Kim, J. C., Kim, M. K., and Sung, Y. K. (2020). Knockdown of FOXA2 Impairs Hair-Inductive Activity of Cultured Human Follicular Keratinocytes. Front. Cel Dev. Biol. 8, 575382. doi:10.3389/ fcell.2020.575382

Bhoopalam, M., Garza, L. A., and Reddy, S. K. (2020). Wound Induced Hair Neogenesis - A Novel Paradigm for Studying Regeneration and Aging. Front. Cel Dev. Biol. 8, 582346. doi:10.3389/fcell.2020.582346

Chen, C.-C., Murray, P. J., Jiang, T. X., Plikus, M. V., Chang, Y.-T., Lee, O. K., et al. (2014). Regenerative Hair Waves in Aging Mice and Extra-follicular Modulators Follistatin, Dkk1, and Sfrp4. J. Invest. Dermatol. 134 (8), 2086-2096. doi:10.1038/jid.2014.139

Chen, Y., Huang, J., Liu, Z., Chen, R., Fu, D., Yang, L., et al. (2020). miR-140-5p in Small Extracellular Vesicles from Human Papilla Cells Stimulates Hair Growth by Promoting Proliferation of Outer Root Sheath and Hair Matrix Cells. Front. Cel Dev. Biol. 8, 593638. doi:10.3389/fcell.2020.593638

Chuong, C.-M. (2007). New Hair from Healing Wounds. Nature 447 (7142), 265-266. doi:10.1038/447265a

Daszczuk, P., Mazurek, P., Pieczonka, T. D., Olczak, A., Boryń, Ł. M., and Kobielak, K. (2020). An Intrinsic Oscillation of Gene Networks inside Hair Follicle Stem laxity, sagging, and rough-textured appearance (Harn et al., 2021). The experimental discoveries and perspectives presented in this Research Topic add new layers for understanding hair follicle stem cell regeneration during aging. With cutting-edge experimental methodologies and everincreasing cross-discipline approaches employed in this Research Topic, we witness the robust progress in this field. We hope this Research Topic will pave new ways for elucidating new mechanisms of aging-related hair follicle stem cell regeneration, and also inspire the development of new therapeutic strategies for treating aging-related hair disorders with this momentum.

\section{AUTHOR CONTRIBUTIONS}

All authors listed have made substantial contributions to the Research Topic, and approved it for publication.

\section{FUNDING}

ML is supported by National Natural Science Foundation of China (82003384), Fundamental Research Funds for the Central Universities (2020CDJYGSG003), Chongqing Talents: Exceptional Young Talents Project (cstc2021ycjh-bgzxm0197), and Scientific Research Foundation from Chongqing University (02210011044110), China. S-JL is supported by Taiwan National Health Research Institutes (NHRI-EX110-10811EI), Taiwan Ministry of Science and Technology (110-2314-B-002-190-MY3) and National Taiwan University Hospital (111IF0006, 110-S5094). C-MC is supported by NIH R01 AR047364, AR060306, and GM125322.

Cells: An Additional Layer that Can Modulate Hair Stem Cell Activities. Front Cel Dev. Biol. 8, 595178. doi:10.3389/fcell.2020.595178

Fukuyama, M., Tsukashima, A., Kimishima, M., Yamazaki, Y., Okano, H., and Ohyama, M. (2021). Human iPS Cell-Derived Cell Aggregates Exhibited Dermal Papilla Cell Properties in In Vitro Three-Dimensional Assemblage Mimicking Hair Follicle Structures. Front. Cel Dev. Biol. 9, 590333. doi:10.3389/ fcell.2021.590333

Gong, L., Xiao, J., Li, X., Li, Y., Gao, X., and Xu, X. (2020). IL-36a Promoted Wound Induced Hair Follicle Neogenesis via Hair Follicle Stem/ Progenitor Cell Proliferation. Front. Cel Dev. Biol. 8, 627. doi:10.3389/ fcell.2020.00627

Harn, H. I.-C., Chen, C.-C., Wang, S.-P., Lei, M., and Chuong, C.-M. (2021). Tissue Mechanics in Haired Murine Skin: Potential Implications for Skin Aging. Front. Cel Dev. Biol. 9, 635340. doi:10.3389/fcell.2021.635340

Ito, M., Yang, Z., Andl, T., Cui, C., Kim, N., Millar, S. E., et al. (2007). Wntdependent De Novo Hair Follicle Regeneration in Adult Mouse Skin After Wounding. Nature 447 (7142), 316-320. doi:10.1038/nature05766

Jang, S., Ohn, J., Kang, B. M., Park, M., Kim, K. H., and Kwon, O. (2020). "Two-Cell Assemblage" Assay: A Simple In Vitro Method for Screening Hair GrowthPromoting Compounds. Front. Cel Dev. Biol. 8, 581528. doi:10.3389/ fcell.2020.581528

Karisma, V. W., Wu, W., Lei, M., Liu, H., Nisar, M. F., Lloyd, M. D., et al. (2021). UVA-triggered Drug Release and Photo-Protection of Skin. Front. Cel Dev. Biol. 9, 598717. doi:10.3389/fcell.2021.598717

Lei, M., and Chuong, C.-M. (2016). Aging, Alopecia, and Stem Cells. Science 351 (6273), 559-560. doi:10.1126/science.aaf1635 
Lei, M., Schumacher, L. J., Lai, Y.-C., Juan, W.-T., Yeh, C.-Y., Wu, P., et al. (2017). Self-organization Process in Newborn Skin Organoid Formation Inspires Strategy to Restore Hair Regeneration of Adult Cells. Proc. Natl. Acad. Sci. USA 114 (34), E7101-E7110. doi:10.1073/pnas.1700475114

Liu, Q., Shi, X., Zhang, Y., Huang, Y., Yang, K., Tang, Y., et al. (2020). Increased Expression of Zyxin and its Potential Function in Androgenetic Alopecia. Front. Cel Dev. Biol. 8, 582282. doi:10.3389/fcell.2020.582282

Matsumura, H., Mohri, Y., Binh, N. T., Morinaga, H., Fukuda, M., Ito, M., et al. (2016). Hair Follicle Aging Is Driven by Transepidermal Elimination of Stem Cells via COL17A1 Proteolysis. Science 351 (6273), aad4395. doi:10.1126/ science.aad4395

Morgun, E. I., and Vorotelyak, E. A. (2020). Epidermal Stem Cells in Hair Follicle Cycling and Skin Regeneration: A View from the Perspective of Inflammation. Front. Cel Dev. Biol. 8, 581697. doi:10.3389/fcell.2020.581697

Qiu, W., Chuong, C. M., and Lei, M. (2019). Regulation of Melanocyte Stem Cells in the Pigmentation of Skin and its Appendages: Biological Patterning and Therapeutic Potentials. Exp. Dermatol. 28 (4), 395-405. doi:10.1111/ exd.13856

Qiu, W., Gu, P.-R., Chuong, C.-M., and Lei, M. (2020). Skin Cyst: A Pathological Dead-End with a New Twist of Morphogenetic Potentials in Organoid Cultures. Front. Cel Dev. Biol. 8, 628114. doi:10.3389/fcell.2020.628114

Suo, L., VanBuren, C., Hovland, E. D., Kedishvili, N. Y., Sundberg, J. P., and Everts, H. B. (2021). Dietary Vitamin A Impacts Refractory Telogen. Front. Cel Dev. Biol. 9, 571474. doi:10.3389/fcell.2021.571474

Wang, B., Yan, S., Yi, Y., Huang, Y., Deng, Z., Zhang, Y., et al. (2020). Purified Vitexin Compound 1 Inhibits UVA-Induced Cellular Senescence in Human Dermal Fibroblasts by Binding Mitogen-Activated Protein Kinase 1. Front. Cel Dev. Biol. 8, 691. doi:10.3389/fcell.2020.00691

Weber, E. L., Lai, Y.-C., Lei, M., Jiang, T.-X., and Chuong, C.-M. (2020). Human Fetal Scalp Dermal Papilla Enriched Genes and the Role of R-Spondin-1 in the Restoration of Hair Neogenesis in Adult Mouse Cells. Front. Cel Dev. Biol. 8, 583434. doi:10.3389/fcell.2020.583434

Wen, L., Miao, Y., Fan, Z., Zhang, J., Guo, Y., Dai, D., et al. (2021). Establishment of an Efficient Primary Culture System for Human Hair Follicle Stem Cells Using the Rho-Associated Protein Kinase Inhibitor Y-27632. Front. Cel Dev. Biol. 9, 632882. doi:10.3389/fcell.2021.632882

Yang, T., Tan, Y., Zhang, W., Yang, W., Luo, J., Chen, L., et al. (2020). Effects of ALA-PDT on the Healing of Mouse Skin Wounds Infected with Pseudomonas aeruginosa and its Related Mechanisms. Front. Cel Dev. Biol. 8, 585132. doi:10.3389/fcell.2020.585132

Yu, N., Hu, T., Yang, H., Zhang, L., Song, Q., Xiang, F., et al. (2020). Twist1 Contributes to the Maintenance of Some Biological Properties of Dermal Papilla Cells In Vitro by Forming a Complex with Tcf4 and $\beta$-Catenin. Front. Cel Dev. Biol. 8, 824. doi:10.3389/fcell.2020.00824

Zhang, C., Wang, D., Wang, J., Wang, L., Qiu, W., Kume, T., et al. (2021). Escape of Hair Follicle Stem Cells Causes Stem Cell Exhaustion During Aging. Nat. Aging 1, 889-903. doi:10.1038/s43587-021-00103-w

Zhang, Y., Huang, J., Fu, D., Liu, Z., Wang, H., Wang, J., et al. (2021). Transcriptome Analysis Reveals an Inhibitory Effect of DihydrotestosteroneTreated 2D- and 3D-Cultured Dermal Papilla Cells on Hair Follicle Growth. Front. Cel Dev. Biol. 9, 724310. doi:10.3389/fcell.2021.724310

Conflict of Interest: The authors declare that the research was conducted in the absence of any commercial or financial relationships that could be construed as a potential conflict of interest.

Publisher's Note: All claims expressed in this article are solely those of the authors and do not necessarily represent those of their affiliated organizations, or those of the publisher, the editors and the reviewers. Any product that may be evaluated in this article, or claim that may be made by its manufacturer, is not guaranteed or endorsed by the publisher.

Copyright (C) 2021 Lei, Lin and Chuong. This is an open-access article distributed under the terms of the Creative Commons Attribution License (CC BY). The use, distribution or reproduction in other forums is permitted, provided the original author(s) and the copyright owner(s) are credited and that the original publication in this journal is cited, in accordance with accepted academic practice. No use, distribution or reproduction is permitted which does not comply with these terms. 